\title{
Comment l'information à transmettre influence-t-elle les constructions référentielle et syntaxique du commentaire sportif en direct?
}

\author{
Augendre, S. \\ UMR5263 CLLE-ERSS (ERSSàB), Département de Sciences du Langage, Université Michel de Montaigne \\ Bordeaux 3 \\ augendre.sandra@wanadoo.fr \\ Mathon, C. \\ EA4195 TELEM (TELANCO), Département de Sciences du Langage, Université Michel de Montaigne Bordeaux 3 \\ catherine.mathon@u-bordeaux3.fr
}

\section{Introduction}

Le travail que nous proposons vise à analyser la construction de la structure référentielle dans un type particulier de corpus oral : le commentaire sportif en direct.

Précisons avant toute chose le cadre dans lequel intervient notre analyse de la structure référentielle. Nous distinguons comme Deulofeu (1999) deux périodes dans le commentaire sportif : «il convient [...] de différencier les moments « d'action », où les journalistes décrivent l'action qui se déroule, des moments de commentaire où les journalistes proposent une analyse de la situation » (Deulofeu, 1999). La première partie du texte global consiste donc en la description des phases de jeu telles qu'elles sont en train de se dérouler sous les yeux du téléspectateur et du commentateur alors que la seconde est en fait du commentaire qui porte soit sur l'action de jeu qui vient de se dérouler, soit sur certains aspects moins immédiats du match et de son contexte (constitution des équipes, rappel de matchs antérieurs, commentaires sur les stratégies de jeu...). Nous nous intéressons ici uniquement aux périodes descriptives du commentaire sportif en direct, discours produit de manière simultanée à l'action qui se déroule sur le terrain, sous les yeux du locuteur (pour une discussion sur la synchronisation du « dire » et du " faire » dans le discours sportif en direct, voir Bres, 2007 : 84-86). Au-delà du caractère motivé de la parole (Boulakia \& Mathon, 2009), le discours descriptif s'adapte dans sa structure à cette situation d'énonciation particulière. En effet, la description simultanée de l'action sur le terrain amène le locuteur à fournir une information qui se résumerait ainsi : un joueur et une action. Cette succession chronologique de désignations des joueurs et des actions forme la matière constitutive du discours descriptif dans le commentaire sportif. Pour autant, il ne s'agit pas d'une simple énumération d'éléments qui font l'action en cours; il y a en effet construction d'une structure référentielle tout au long du discours et dans chacune des périodes descriptives qui participe à la cohérence du discours.

Nous commencerons par présenter nos hypothèses quant aux relations entretenues entre la transmission de l'information portée par le discours descriptif, la référence et la réalisation des expressions linguistiques référentielles. Dans un deuxième temps, nous introduirons les données relatives au corpus et aux annotations et analyses réalisées. Nous passerons ensuite à l'analyse des résultats obtenus pour les différents paramètres considérés et terminerons par une illustration sur les séquences Prénom + Nom du corpus.

\section{Hypothèses}

Le thème de la structure référentielle, mis en relation avec un type particulier de discours, les phases descriptives d'un commentaire sportif, nous a conduites à établir les deux pistes de travail suivantes : 
1) Quels sont les paramètres pertinents pour décrire la structure référentielle d'un commentaire sportif ?

Il s'agit ici de voir si la construction de la structure référentielle au sein du commentaire sportif est oui ou non spécifique à ce type de discours, mais également d'identifier les propriétés des expressions référentielles dans le commentaire ou encore de définir des types de construction référentielle...

2) Quel rôle joue l'iconicité dans la construction de la structure référentielle du commentaire sportif ?

Dans le prolongement du questionnement précédent, il nous semble probable que l'iconicité induite par la situation de parole ait une influence sur l'organisation de l'information dans le discours descriptif. La construction des référents, qui constituent une part importante de l'information transmise dans le discours, et les constructions syntaxiques sous-jacentes à l'introduction des référents, vont donc être motivés par la situation de parole.

\section{Corpus et méthodologie}

Dans cette partie, nous allons d'abord présenter le corpus et les unités sur lesquelles vont porter notre analyse, les expressions référentielles. Nous introduirons ensuite les notions qui seront utilisées dans la recherche, le cadre dans lequel elle s'inscrit et enfin l'ensemble des paramètres observés sur les données recueillies.

\subsection{Le corpus}

Nous travaillons sur le commentaire télévisé d'un match de rugby, enregistré lors de la Coupe du Monde de Rugby de septembre 2007, et qui fait partie d'un corpus beaucoup plus important, composé d'une part de commentaires sportifs télévisés récoltés lors de cette compétition en français principalement, et d'autre part d'enregistrements de supporters en train d'assister à ces rencontres (Lortal \& Mathon, 2008). Pour cette étude, nous nous sommes intéressées au commentaire journalistique uniquement et nous nous sommes concentrés sur la rencontre France-Argentine, qui a inauguré cette compétition, commentée en français. Cet enregistrement a fait l'objet d'une transcription orthographique effectuée à l'aide du logiciel Transcriber. Le fichier sonore et la transcription ont été ensuite alignés avec le logiciel WinPitch Pro (Martin, 2000). Cet enregistrement est d'une durée totale de 1 heure et 48 minutes et se présente sous la forme d'un fichier en format .wav $(22050 \mathrm{~Hz}, 16$ bits, mono). Trois locuteurs se partagent de manière inégale le temps de parole au cours de cet enregistrement.

Le tableau ci-dessous montre la répartition du temps de parole entre ces trois locuteurs :

\begin{tabular}{|c|c|}
\hline Temps de parole locuteur 1 & $40 \mathrm{mn}$ \\
\hline Temps de parole locuteur $\mathbf{2}$ & $13 \mathrm{mn}$ \\
\hline Temps de parole locuteur 3 & $2 \mathrm{mn}$ \\
\hline Temps de parole total & $55 \mathrm{mn}$ \\
\hline Durée d'enregistrement & $108 \mathrm{mn}$ \\
\hline
\end{tabular}

Tableau 1 : Répartition des temps de parole des locuteurs en minutes

C'est le locuteur 1, en tant que commentateur principal, qui intervient le plus longuement dans cet enregistrement et c'est sur le discours de ce locuteur que porteront plus particulièrement nos analyses. En effet, c'est à lui qu'appartient de faire la description en direct de l'action qui se déroule sur le terrain de jeu. 


\subsection{Identification des périodes descriptives}

La première tâche que nous avons effectuée a été de distinguer les périodes descriptives des périodes de commentaires. Cette annotation a été faite manuellement par les auteurs, avec écoute du signal. L'annotation a d'abord été réalisée de manière séparée par les auteurs, qui ont ensuite confronté et harmonisé leur découpage.

L'isolement des périodes descriptives a été effectué avec le logiciel Transcriber : au début et à la fin de chaque période descriptive, une balise de section a été créée pour marquer les limites de la période.

Au total, nous avons obtenu 120 périodes descriptives de tailles variables, au sein desquelles nous avons travaillé sur les expressions linguistiques référentielles.

\subsection{Identification et annotation des référents (ID, IP et RPn)}

Pour ce travail, nous avons choisi de reprendre la distinction qu'ont déjà proposée des linguistes tels que Chafe (1976 : 30), Prince (1981:226) ou Lambrecht (1988:144), de trois types de référents :

- les référents activés pour la première fois («newly activated ») ou inactifs (« unactivated ») : ils sont introduits pour la première fois dans l'univers de discours

- les référents accessibles («accessible ») : ils sont introduits pour la première fois dans une période descriptive et récupérables dans le discours précédent

- les référents (déjà) activés (« (already) activated ») : il s'agit des expressions coréférentes présentes dans chaque période descriptive

Dans un premier temps, nous avons distingué le niveau du discours et le niveau de la période descriptive en isolant les référents introduits pour la première fois dans le discours (ID, Introduction-Discours) et les référents introduits pour la première fois dans une période descriptive (IP, Introduction-Période). Cette distinction a été introduite dans l'étude dans le seul but de pouvoir confirmer ou infirmer l'hypothèse 1, selon laquelle l'expression référentielle ne dispose pas des mêmes propriétés quand son référent est introduit pour la première fois dans l'univers de discours et quand son référent est introduit pour la première fois dans une période descriptive, en ayant déjà été introduit au niveau du discours.

Dans un deuxième temps, au sein de chaque période descriptive, nous avons isolé les expressions coréférentielles et avons distingué la première mention du référent (IP, Introduction-Période) et la ou les éventuelles reprises présentes dans le discours suivant proche (RP1, RP2, RP3..., Reprise-Période).

Dans l'ensemble des périodes descriptives du commentaire analysé, 40 référents (humains) sont introduits sous la forme de noms propres : il s'agit des noms des joueurs, de l'arbitre, de l'entraîneur, des équipes...

Ces référents font l'objet de 510 activations, dans le discours (ID, 40 cas) et dans ses périodes descriptives (IP, 470 cas), et de 346 reprises (RP) sous des formes différentes, essentiellement des pronoms relatifs sujets (30\% des reprises), des anaphores nominales fidèles $(28 \%)$ et des clitiques sujet $(21 \%)$. 


\begin{tabular}{|c|l|c|}
\hline Type de référent & \multicolumn{1}{c|}{ Exemple } & $\begin{array}{c}\text { Répartition des } \\
\text { reprises dans le } \\
\text { corpus (\%) }\end{array}$ \\
\hline $\begin{array}{c}\text { ID (1ère introduction dans } \\
\text { le discours) }\end{array}$ & $\begin{array}{l}\text { après avoir demandé si tout le } \\
\text { monde était prêt le coup d'envoi de } \\
\text { la coupe du monde 2007 est donné } \\
\text { par David Skrela }\end{array}$ & \\
\hline $\begin{array}{c}\text { IP (1ère introduction dans } \\
\text { une période descriptive) }\end{array}$ & $\begin{array}{l}\text { le renvoi de David Skrela un en- } \\
\text { avant argentin mêlée introduction } \\
\text { française }\end{array}$ & $30 \%$ \\
\hline RP Relatif Sujet & $\begin{array}{l}\text { hou et contré David Skrela et qui } \\
\text { commet un en-avant }\end{array}$ & $28 \%$ \\
\hline RP Anaphore nominale \\
fidèle & $\begin{array}{l}\text { Mignoni pour Skrela attention à ne } \\
\text { pas se faire contrer cette fois } \\
\text { impeccable Skrela pour trouver la } \\
\text { touche }\end{array}$ & $21 \%$ \\
\hline RP Clitique Sujet & $\begin{array}{l}\text { et le dégagement de Skrela qu'on } \\
\text { sent très fébrile lui aussi il était très } \\
\text { ému au moment des hymnes } \\
\text { nationaux }\end{array}$ & 2 \\
\hline
\end{tabular}

Tableau 2: Type de référents et répartition des principales catégories de reprises en \%

\subsection{Identification et classement des expressions linguistiques référentielles}

Dans notre étude de la structure référentielle, nous avons choisi de considérer également les différentes réalisations linguistiques de nos référents dans le discours. Nous avons donc annoté ces expressions en termes de :

- catégories lexicales $\rightarrow$ Nom commun (N), Nom Propre (Np), Clitique Sujet (ClS), Clitique Complément (ClC), Relatif Sujet (RelS), Relatif Complément (RelC), Proforme (Prof), Posessif (Poss), pronom Tonique (To) ;

- catégories syntagmatiques $\rightarrow$ Syntagme Nominal (SN), Syntagme Prépositionnel (SP) et les catégories $\mathrm{ClC}, \mathrm{ClS}$, To, RelS, RelC, Poss, Prof ;

- prépositions pour les syntagmes prépositionnels (SP).

A partir du croisement de ces données avec celles relatives aux types de référents (ID-IP, IP et RPn), nous avons la possibilité, entre autres, d'observer la fréquence globale de chaque catégorie ou préposition ainsi que l'utilisation qui est faite de chacune d'entre elles dans le type de discours à l'étude.

Si l'ensemble des données seront présentées dans la partie réservée aux résultats, nous pouvons d'ores et déjà fournir les tendances générales et nos observations préliminaires :

- la catégorie lexicale dominante est le nom propre (Prénom+Nom, Titre+Nom, Nom seul, Prénom/Surnom), en particulier pour une première introduction dans le discours et/ou dans une période : ce type d'expression référentielle représente $58 \%$ des activations et réactivations de référents du corpus ( $76 \%$ des IP et ID-IP et $28 \%$ des RP du corpus sont des Np) ;

- en ce qui concerne les reprises, les catégories dominantes sont les relatifs sujet ( $29 \%$ des cas), les noms propres $(28 \%$ des cas), les pronoms clitiques sujet ( $21 \%$ des cas) et les noms communs $(12 \%$ des cas). L'ensemble des cinq autres catégories (Poss, Prof, To, $\mathrm{ClC}$ et RelC) ne dépassent pas les $10 \%$ en termes de fréquence d'utilisation par le locuteur ; 
- enfin, nous y reviendrons, nous n'avons pas observé de tendances particulières au niveau du choix de la catégorie en fonction de la distance présente entre la première introduction du référent (IP) et les différentes reprises (de RP1 à RP11). Par exemple, on a autant de noms propres que de clitiques sujet dans nos reprises les plus distantes (de RP4 à RP11).

\subsection{Contextes gauche et droit des expressions référentielles}

Nous avons fait dans un premier temps la distinction entre les référents introduits par une préposition et ceux qui étaient introduits de manière directe. Parmi les prépositions introduisant les référents, nous avons distingué celles qui impliquent une dépendance à l'élément directement à gauche de celles qui ne marquent pas nécessairement de dépendance syntaxique forte.

On retrouve principalement à gauche des référents les quatre prépositions suivantes :

\begin{tabular}{|c|c|}
\hline avec & $14 \%$ \\
\hline de & $9,5 \%$ \\
pour & $9 \%$ \\
\hdashline par & $5,5 \%$ \\
\hline
\end{tabular}

Tableau 3 : Répartition en \% des principales prépositions susceptibles d'introduire des référents

Les prépositions avec et pour ne sont pas nécessairement induites par les éléments à gauche, et très souvent, elles ne marquent pas de dépendance du référent. En revanche, la préposition de marque souvent une dépendance à un noyau nominal, tandis que la préposition par implique une dépendance à une forme verbale passive ou participiale.

Nous avons dans un second temps annoté systématiquement la catégorie morpho-syntaxique de l'élément directement à gauche et celle de l'élément directement à droite du référent. Cette annotation va nous permettre de prédire les possibles dépendances syntaxiques à gauche et à droite du référent et constitue donc une aide supplémentaire dans l'analyse des expressions référentielles en contexte.

\subsection{Annotation macrosyntaxique}

Afin de rendre compte des expressions référentielles du commentaire sportif, nous avons choisi d'introduire la notion de «catégories macrosyntaxiques », en considérant, dans la lignée de BlancheBenveniste (1997: 111), que « la syntaxe de la phrase et des propositions, fondée sur les catégories grammaticales et leurs fonctions, ne suffit pas à rendre compte de certaines organisations de la langue parlée ».

Le principe de l'analyse macrosyntaxique, qui permet d'aller au-delà des relations de dépendance, se résume comme suit: «les unités macrosyntaxiques sont établies par un découpage des énoncés s'appuyant notamment sur des critères prosodiques, sur la portée des modalités d'énoncé et sur des relations de dépendance syntagmatique » (Deulofeu, 1999).

Comme Deulofeu (1999), nous avons observé dans le corpus, la part très importante des constructions averbales dans les périodes descriptives. C'est en grande partie pour cette raison que nous adoptons, tout comme lui, une analyse macrosyntaxique des énoncés, propre à expliciter les constructions averbales, qu'elles soient constituées d'une expression référentielle seule (par exemple : //David Skrela//) ou d'une expression référentielle associée à d'autres éléments (par exemple : //avec Rémy Martin// ; //avantage laissé à Mario Ledesma// ; //Felipe Contepomi pour ce drop//...). De ce fait, nous avons distingué deux types de relations entre le référent et le macrosyntagme : soit le référent appartient au macrosyntagme, 
en est un élément constitutif avec d'autres éléments, soit le référent constitue à lui tout seul le macrosyntagme.

Nous avons appliqué les catégories macrosyntaxiques telles qu'elles sont définies par Blanche-Benveniste (1997): préfixe, postfixe, noyau, suffixe. Ces catégories sont définies en fonction de critères de dépendance ou d'indépendance sémantique, syntaxique et prosodique (Blanche-Benveniste, 1997; Martin, 2009...). Pour aider à définir ces macrosyntagmes et la place des référents par rapport à ceux-ci, nous avons donc réalisé d'une part une annotation des contextes gauche et droite des expressions référentielles, et d'autre part une analyse prosodique des groupes macrosyntaxiques.

\subsection{Analyse prosodique}

Nous avons conduit une analyse prosodique en prenant essentiellement en compte les mesures de débit, les registres de voix et la variation mélodique. Cette analyse nous a tout d'abord aidées lors de l'identification des macrosyntagmes, en mettant en lumière des relations de dépendance ou de nondépendance entre les éléments: «en l'absence de relations de dépendance aux frontières, c'est la structure prosodique qui va assurer la cohérence de l'énoncé » (Martin, 2009 : 124). Afin de mesurer le degré d'indépendance du segment par rapport à son contexte immédiat, nous avons donc systématiquement annoté la présence d'une pause directement à gauche et/ou à droite des expressions référentielles du corpus.

Notre motivation première à ce niveau d'analyse est de montrer que la prosodie peut être un révélateur de l'état d'activation du référent (Baumann \& Riester, 2010).

\section{Résultats}

Après avoir fourni l'ensemble des données relatives aux objectifs de ce travail ainsi qu'aux notions et méthodes que nous avons choisies d'utiliser, nous allons présenter nos résultats, selon l'organisation thématique suivante: nous fournirons et analyserons d'abord les données relatives aux expressions linguistiques et à leurs référents, puis détaillerons et exemplifierons les différentes structures référentielles disponibles dans le corpus. Enfin, nous proposerons d'évaluer l'influence qu'a l'iconicité sur les structures informationnelle, référentielle et syntaxique des périodes descriptives d'un commentaire sportif.

\subsection{Types de référents et d'expressions linguistiques : croisement des données}

Une première analyse des données nous amène à observer que ce n'est pas au niveau des réalisations linguistiques que la structure référentielle du commentaire sportif en direct est spécifique. Le tableau 3 montre la répartition en \% des nouveaux référents (de discours et de période) et de leurs reprises en fonction de la catégorie lexicale des expressions linguistiques correspondantes :

\begin{tabular}{|c|c|c|c|}
\hline Catégories lexicales $\quad$ Types de référents & ID-IP & IP & RPn \\
\cline { 2 - 3 } Relatif sujet (RelS) & $0 \%$ & $0 \%$ & $30 \%(104)$ \\
\hline Nom propre (Np) & $87 \%(35)$ & $77 \%(363)$ & $28 \%(96)$ \\
\hline Clitique sujet (ClS) & $3 \%(1)$ & $1 \%(4)$ & $21 \%(71)$ \\
\hline Nom commun (N) & $10 \%(4)$ & $22 \%(102)$ & $12 \%(40)$ \\
\hline
\end{tabular}




\begin{tabular}{|c|c|c|c|}
\hline Catégories lexicales $\quad$ Types de référents & ID-IP & IP & RPn \\
\cline { 1 - 3 } Possessif (Poss) & $0 \%$ & $0 \%$ & $3 \%(11)$ \\
\hdashline Proformes (Prof) & $0 \%$ & $0 \%(1)$ & $3 \%(10)$ \\
\hline Pronom tonique (To) & $0 \%$ & $0 \%$ & $2 \%(8)$ \\
\hline Clitique complément (ClC) & $0 \%$ & $0 \%$ & $1 \%(5)$ \\
Relatif COD (RelC) & $0 \%$ & $0 \%$ & $0 \%(1)$ \\
\hline
\end{tabular}

Tableau 4: Répartition (en \%) des référents du corpus par catégorie morpho-syntaxique

Comme on pouvait s'y attendre, les référents sont massivement introduits, aussi bien au niveau du discours (ID-IP) qu'au niveau de la période descriptive (IP), sous la forme de syntagmes nominaux (SN) dont la tête est un nom propre (dans $82 \%$ des cas, ID-IP et IP confondus) ou un nom commun (dans $16,5 \%$ des cas, ID-IP et IP confondus).

Les reprises (RP) sont elles aussi attendues : quand il ne s'agit pas d'anaphores nominales fidèles ou infidèles (le RP est un nom propre dans $28 \%$ des cas et une description définie dans $12 \%$ des cas), les référents sont essentiellement repris par un/des relatif/s ou clitique/s sujet (respectivement dans $30 \%$ et $21 \%$ des cas).

\subsection{Les principales structures référentielles du corpus}

L'analyse des données nous a permis de distinguer différents types de construction de la chaîne référentielle dans une période descriptive. Nous allons présenter ci-dessous un exemple détaillé pour les configurations les plus récurrentes dans le corpus :

\subsubsection{Cas "prototypique ": la première introduction d'un référent inactif dans le discours ou dans une période se fait par un nom propre et les éventuelles reprises appartiennent à la catégorie pronominale}

Il s'agit de la configuration la plus récurrente du corpus : le locuteur active un référent avec un nom propre dans $82 \%$ des cas (ID-IP et IP confondus) et le met en lien avec une ou plusieurs actions (étapes de l'action), engendrant la présence d'expressions coréférentes (RP) dans le contexte droit proche, des pronoms dans $57 \%$ des cas.

Prenons quelques exemples de ce premier type d'organisation référentielle :

1. Prénom + Nom + RelS + RelS :

hé oui dans les vingt-deux mètres français remise en jeu Ledesma // et Rémy Martin qui vient batailler encore // et qui gagne ce ballon pour euh Ibanez

2. Nom $+\mathrm{ClS}+\mathrm{ClS}$ :

avec Heymans il a des appuis de feu il préfère jouer au pied

3. Nom $(+\mathrm{To})+\mathrm{ClS}$ (dislocation à gauche) :

et là Mignoni lui il a obtenu la pénalité

L'association du $\mathrm{Np}$ et du clitique sujet dans l'exemple 3 illustre un cas de dislocation à gauche : l'expression référentielle directe (le $\mathrm{Np}$ ) est placée à l'initiale, en position de préfixe (cf. BlancheBenveniste, 1997 : 118), puis est reprise par un pronom clitique coréférent qui fait le lien entre le référent isolé à l'initiale et l'action présentée par la suite. 
Les trois extraits ci-dessus illustrent le type de séquences IP + RPn le plus représenté dans les PD, qu'il est possible de formaliser comme suit :

$\mathrm{IP}(\mathrm{Np})+\mathrm{RP} 1(\mathrm{Pr})+\mathrm{RP} 2(\mathrm{Pr}) \ldots$

\subsubsection{Cas de « rupture de la chaîne » : un référent est réactualisé par un nom propre}

Dès que l'action change d'agent, il y a introduction de nouveaux référents dans le discours (eux aussi sous la forme de noms propres éventuellement accompagnés de coréférents pronominaux). Si un référent doit être repris dans la même période descriptive alors que d'autres référents ont été introduits entre sa première introduction dans la période et sa reprise, le locuteur évite les pronoms et favorise la compréhension en utilisant plutôt une anaphore directe, c'est-à-dire la réactualisation du référent par le nom propre.

Voici deux exemples de cette séquence activation + rupture + réactualisation :

4. Nom1 + RelS1 + C1S1 + Noms $2 / 3 / 4 / 5+$ Nom1 + Nom $6+$ Nom1

alors c'est pas Elissalde qui va se charger de ce renvoi il va aller derrière ses avants derrière les \{initelligible\} // Traille pour un dégagement très lointain sans doute voilà qui est fait // pour aller tout de suite dans le camp argentin // ballon* récupéré par Borgès pour // Hernandez \{initelligible\} // ouais alors Harinordoquy / bien // et quelle belle prise de balle / c'est bien / ah ça c'est beau // superbe // Elissalde / et y'a des gros là-bas ils vont / avec Michalak // ouais \{initelligible\} // Elissalde encore pour Dominici // allez

Dans la première partie de l'extrait, il n'est question que du joueur Elissalde et la séquence d'expressions référentielles est «prototypique» (cf. cas A.) : le joueur est introduit dans le discours avec son nom (Nom1), puis repris par des pronoms (RelS1 et ClS1).

Dans un deuxième temps, en lien avec l'action, quatre nouveaux référents sont introduits dans la période descriptive (sous forme de nom propre également, Noms2/3/4/5).

Dans un troisième temps, le joueur 1, Elissalde, revient au cœur de l'action : le locuteur doit réintégrer cet agent dans son discours et choisit l'expression directe et référentiellement autonome pour le faire, le nom propre.

En fin d'extrait, nous avons un deuxième exemple de gestion de la rupture référentielle par l'anaphore directe, avec la séquence Elissalde + // des gros $+i l s+$ Michalak // + Elissalde. L'introduction de référents $\mathrm{X}$ et $\mathrm{Y}$ entre deux mentions d'un référent $\mathrm{Z}$ conduit, indépendamment de l'appartenance de ces deux expressions à la même période descriptive, à un retour à la forme prototypique de l'IP pour $\mathrm{X}$, le nom propre.

Cette deuxième configuration peut être représentée de la façon suivante :

IP1 $(\mathrm{Np} 1)+\mathrm{RP} 1(\mathrm{Pr})+\mathrm{IP} 2(\mathrm{~N}(\mathrm{p}) 2)+\mathrm{RP} 1(\mathrm{~Np})+\mathrm{RP} 2(\mathrm{~Np}) \ldots$

\subsubsection{L'introduction du référent par un nom propre est retardée pour un effet d'emphase}

Dans certains cas, le référent n'est pas introduit dans la période par son nom mais est d'abord activé par une description définie, éventuellement reprise par une ou d'autres expressions coréférentes (cf. exemple ci-dessous). Le nom propre, qui permet de figer la référence, est alors précédé dans le discours par des expressions coréférentes, ce qui crée un effet d'attente et une mise en focus du référent et de son identification, éventuellement renforcée par des marques syntaxiques (et/ou prosodiques) :

$\mathrm{IP}(\mathrm{N})+\mathrm{RP} 1(\mathrm{Pr})+\ldots+\mathrm{RPn}(\mathrm{Np})$

Dans l'exemple 5 ci-dessous, nous avons un séquence de trois expressions coréférentes : un syntagme nominal (le buteur de l'équipe de France), un pronom tonique (lui) et un ensemble Prénom + Nom 
(David Skrela). En plus de la coprésence et de la proximité de trois mentions du même référent, la syntaxe renforce ici la proéminence attribuée par le locuteur au joueur : l'extrait présente une dislocation à gauche de la description définie ainsi que deux structures présentatives en " c'est X », qui conduisent à l'isolation des expressions linguistiques et à la focalisation de leurs référents.

5. $\mathrm{SN}+\mathrm{To}+\mathrm{Np}$

le buteur de l'équipe de France / c'est lui / c'est David Skrela // un peu moins de vingt-six mètres la ligne que vous voyez devant lui // c'est la ligne des vingt-deux mètres mais avec euh// le décalage // ça fait un peu moins de vingt-six mètres // pour égaliser // concentration de David Skrela // et trois partout

\subsubsection{L'introduction du référent par un nom propre est ajoutée en fin d'énoncé pour clarifier le propos}

Nous avons également de nombreux cas de dislocations à droite de noms propres qui ne répondent pas à un besoin d'emphase mais visent plutôt à clarifier le propos, comme dans l'exemple suivant :

6. Nom + ClS + Prénom+Nom

Fernandez Lobbe a très bien pris ce ballon // sur le renvoi français // et Hernandez // ne trouvera pas la touche il est tombé sur Harinordoquy // Heymans // il va taper loin devant Cédric Heymans

Dans un premier temps, le locuteur introduit l'IP Heymans, qui constitue un énoncé en soi, un noyau. Nous profitons de cet exemple pour mentionner le fait que le corpus contient beaucoup de ces énoncés averbaux constitués du seul nom propre du joueur, qui signalent le nom du joueur qui détient (même pour un court instant) le ballon.

Dans un deuxième temps, le locuteur donne une précision supplémentaire sur l'action menée par le référent (taper loin devant). L'information assertée est uniquement l'action, pas le joueur, qui appartient à l'arrière-plan informationnel. Dans ce nouvel énoncé, l'agent est d'abord repris par un clitique sujet à l'initiale $(i l)$, puis rappelé sous la forme d'une anaphore directe de type Prénom + Nom (Cédric Heymans) en finale, non pas afin de permettre l'identification du référent (qui est déjà établie dans le discours précédent), mais pour s'assurer qu'il n'y a pas confusion sur le référent de ce clitique, autrement dit pour clarifier le propos :

$\mathrm{IP}(\mathrm{Np})+\mathrm{RP} 1(\mathrm{Pr})+\mathrm{RP} 2(\mathrm{~Np}) \ldots$

\subsection{Influence de l'iconicité sur la structure informationnelle, sur la structure référentielle qui en découle et sur les constructions syntaxiques afférentes}

Nous l'avons dit dans l'introduction, ce qui fait la spécificité du commentaire sportif en direct, c'est l'iconicité qu'il présente, dans les périodes descriptives principalement : le discours se doit de suivre le rythme des actions qui se déroulent sur le terrain de jeu.

Par conséquent, l'iconicité influence la structure informationnelle du discours, au sens où c'est de ce qui se passe sur le terrain que dépendent les informations qui vont être données par le locuteur. Ces informations sont en général de deux types :

- Qui ? : Introduction des référents(-joueurs)

- Quoi ? : Attribution d'actions de jeu aux référents(-joueurs)

La structure informationnelle du commentaire en direct, le fait que cette structure se résume à donner deux types d'informations, va influencer la structure référentielle, puisque les référents constituent une bonne part de l'information donnée par le locuteur.

L'analyse des données nous a conduites à distinguer trois organisations de la structure informationnelle, détaillées ci-dessous, mais également à observer que la structure référentielle et les expressions linguistiques dépendent en grande partie de ces trois configurations. 


\subsubsection{Le commentateur indique le joueur puis introduit l'action associée}

On trouve dans cette première configuration informationnelle beaucoup d'énoncés à tête nominale, constitués de la façon suivante : Référent + Reprise (relatif ou clitique) $+\mathbf{S V}$.

Voici deux exemples tirés du corpus :

7. (avec un relatif) :

c'est une touche pour l'Argentine / avec Traille qui saigne hein

8. (avec un clitique) :

que dit Monsieur Spreadbury il dit que c'est la fin

A l'inverse, il est à noter que la suite directe Sujet/Agent + Verbe/Action (Référent + SV, cf. ex.9), sans rupture ni rappel du référent, est assez peu fréquente dans le corpus :

9. $\mathrm{Np}+\mathrm{V}$

Rougerie a récupéré

\subsubsection{Le commentateur indique l'action puis introduit le joueur associé}

Dans cette seconde configuration, ce sont les énoncés à tête nominale et prépositionnelle qui sont les plus fréquents. L'action est décrite sous la forme d'un syntagme nominal (SN) ou d'un syntagme prépositionnel (SP) et l'agent est introduit comme complément du nom ( $\mathrm{SP}(\mathrm{de}))$ :

10. et le dégagement de Skrela qu'on sent très fébrile lui aussi

11. allez de suite dans le camp euh /argentin / avec un départ de Roncero

\subsubsection{Le commentateur indique le joueur sans préciser l'action}

La troisième configuration comporte une forme d'implicite dans la façon dont est transmise l'information, puisque celle-ci consiste uniquement dans la nomination des référents. Implicitement, on sait que si les référents sont nommés, c'est qu'ils ont accès au ballon, mais si le locuteur n'en dit pas plus, cela signifie que cet accès a été bref (séquence de passes).

On trouve ce genre de configuration de l'information, quand sur le terrain on assiste à une phase dans laquelle les joueurs essaient de construire leur jeu autour de passes afin de trouver une ouverture dans la défense de l'adversaire. Dans le discours descriptif, ce type de phase se traduit par une succession rapide d'expressions linguistiques indépendantes référentiellement, syntaxiquement et prosodiquement. En d'autres termes: «le commentateur verbalise seulement les actants par leur anthroponyme, en une énumération qui prend fin lorsque l'action elle-même, conduite par les membres d'une seule et même équipe,, se termine, à savoir lorsque les joueurs perdent la balle. » (Bres, 2007 : 87).

À partir de leur structure interne, nous avons distingué deux types de séquences entrant dans ces phases d'énumération de référents, les SN (Prénom+Nom et Nom) et les SP (Prép+Prénom+Nom et Prép+Nom) :

- L'expression référentielle est contenue dans un SN ayant la forme Prénom + Nom ou Nom

12. attention à Leguizamón / Pichot / avec à hauteur et la défense française qui ne bronche pas pour le moment / Pichot encore / à l'extérieur avec Borges

- L’expression référentielle est contenue dans un SP ayant la forme Préposition + Prénom + Nom ou Préposition + Nom

13. les voilà les meilleurs ballons à jouer avec Jauzion / avec Rémy Martin / oh les jeux / avec Pieter de Villiers / avec Heymans maintenant c'est la grande relance / française jusqu'à Rougerie 
La préposition avec est la préposition la plus souvent employée devant un référent $\mathrm{Np}$ : 15\% pour les formes Nom, et $9 \%$ pour les formes Prénom+Nom. Elle n'est généralement pas régie par une forme verbale ou nominale à gauche et ne sert pas à établir une relation mais seulement à introduire le référentjoueur qui a momentanément le ballon. Ces SP apparaissent dans le discours comme des noyaux indépendants, au niveau syntaxique mais également au niveau prosodique, les séquences avec $+\mathrm{P}+\mathrm{Np}$ et avec $+\mathrm{N}$ étant souvent précédées et suivies d'une pause.

\section{Application à la catégorie $\mathrm{P}+\mathrm{Np}$}

Afin d'illustrer nos analyses, notamment les différentes configurations observées en termes de progression de l'information, de référence et de formes syntaxiques, nous allons nous intéresser à un seul type d'expression référentielle : les $\mathrm{Np}$ sous la forme $\mathrm{P}+\mathrm{Np}$

Notre corpus contient un nombre très important de $\mathrm{Np}$ (494), qui représentent $82 \%$ des (ID-) IP du corpus (398/510) et $28 \%$ des RP (96/346). Ces Np se présentent essentiellement sous deux formes :

Nom de famille seul $(83 \%$ des $\mathrm{Np})$

Prénom + Nom $(16 \%$ des Np)

Nous présentons ci-dessous les caractéristiques des séquences $\mathrm{P}+\mathrm{Np}$ en fonction des trois types de progression de l'information que nous avons distingués dans la partie précédente.

\subsection{Configuration informationnelle 1}

Il s'agit de la configuration selon laquelle le commentateur indique le nom du joueur et indique ensuite ce que fait le joueur. La forme la plus fréquente de cette configuration est :

$\mathrm{P}+\mathrm{Np}+$ Reprise (Relatif) + forme verbale

14. hé oui dans les vingt-deux mètres français remise en jeu Ledesma / et Rémy Martin qui vient batailler encore / et qui gagne ce ballon pour euh Ibanez

$10 \%$ des référents $\mathrm{P}+\mathrm{Np}$ sont construits ainsi

Beaucoup moins fréquente est la forme : $\mathbf{P}+\mathbf{N p}+$ forme verbale. Elle ne concerne que $4 \%$ des référents $\mathrm{P}+\mathrm{Np}$.

15. Fernandez Lobbe a très bien pris ce ballon / sur le renvoi français / et Hernandez / ne trouvera pas la touche il est tombé sur Harinordoquy

\subsection{Configuration informationnelle 2}

Il s'agit de la configuration selon laquelle le commentateur indique l'action qui est menée et ensuite le référent-joueur qui fait l'action. On la retrouve sous deux formes :

- SN Action + Préposition $+\mathrm{P}+\mathrm{Np}$

Cette première construction est assez fréquente puisqu'elle concerne $21,5 \%$ des référents $\mathrm{P}+\mathrm{Np}$. Le référent est principalement introduit par la préposition de. Il est précédé d'un SN qui indique l'action.

16. coup de pied tombé pour ce renvoi / qu'on appelle aussi drop / et récupération de Raphaël Ibanez

(Pour une structure syntaxique identique, cf. exemples 10 et 11) 
- SV Action + Préposition par $+\mathrm{P}+\mathrm{Np}$

On observe quelques cas où l'action prend la forme d'un verbe au passif, suivi de la préposition par qui introduit le référent $\mathrm{P}+\mathrm{Np}$.

17. le coup d'envoi de la coupe du monde deux mille sept est donné par David Skrela

- SV (ne désigne pas nécessairement l'action) + Référent-Joueur (postfixe)

18. avec Skrela pour trouver / pas la touche / forcer Nani Corleto à une relance il y excelle Nani Corleto une chandelle

(Pour un exemple structurellement similaire cf. exemple 6)

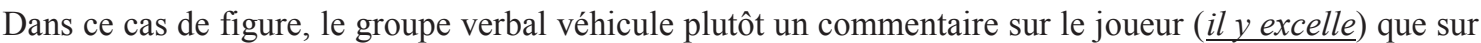
l'action proprement dite, qui est décrite dans le SP pour trouver... qui précède. La position de postfixe de l'anaphore nominale fidèle Nani Corleto permet de fixer la référence du pronom $\mathrm{il}$.

\subsection{Configuration informationnelle 3}

Il s'agit des cas où le fait de nommer le référent suffit à l'apport informationnel, et où les référents constituent des énoncés à part entière. On observe deux formes à cette configuration :

- L'expression P + Np se présente seule

La suite $\mathrm{P}+\mathrm{Np}$ constitue un noyau à elle seule, c'est-à-dire un élément syntaxiquement et prosodiquement indépendant. Par prosodiquement indépendant, on entend notamment qu'il porte une intonation finale d'énoncé. L'indépendance prosodique de l'expression référentielle $\mathrm{P}+\mathrm{Np}$ est également marquée par la présence de pauses à gauche et à droite du référent.

19. attention à ne pas garder le ballon // à le libérer vite // David Skrela // première grosse alerte

(Pour un exemple de Noyau constitué par un Nom Propre seul, cf. exemple 12)

- Une préposition (avec, pour...) précède $\mathrm{P}+\mathrm{Np}$

La séquence $\mathrm{P}+\mathrm{Np}$ est parfois introduite par une préposition et l'ensemble constitue un noyau syntaxiquement et prosodiquement indépendant. Cette construction est restreinte aux prépositions avec et pour. Seules ces deux prépositions peuvent apparaître en introduction du référent, sans impliquer de dépendance syntaxique par rapport à un élément situé à gauche du SP.

20. allez le ballon est argentin avec Alvarez Kairelis / Pichot / et Hernandez hou qui se trompe complètement

Très souvent, ces référents introduits par les prépositions avec ou pour, et qui sont catégorisés comme des noyaux, présentent une pause à gauche $(75 \%$ des cas) et une pause à droite (100\% des cas), qui permettent de les délimiter prosodiquement.

\section{1. ouais mais on va jouer au pied côté français // avec Damien Traille}




\section{Conclusion}

L'étude de l'interface information-référence-syntaxe que nous avons menée sur un corpus de commentaire sportif en direct nous a conduites aux conclusions et réflexions suivantes :

1) La structure référentielle des périodes descriptives a finalement une construction assez classique, qu'on trouverait dans un autre type de discours :

- Là où des reprises pronominales pourraient porter à confusion sur l'identification du référent, le locuteur opte plutôt pour l'anaphore nominale fidèle.

- Lors de l'attribution d'une succession d'actions à un même référent, celui-ci sera introduit sous la forme d'une description définie ou d'un $\mathrm{Np}$ puis repris autant que nécessaire par des expressions pronominales coréférentes

- À partir du moment où l'introduction d'un référent est suivie de celle d'un ou de plusieurs autres référents, le locuteur se doit de réactiver le référent sous une forme directe, comme s'il s'agissait d'un IP ou d'un ID.

- Les catégories morpho-syntaxiques représentées dans le corpus sont, pour les introductions comme pour les reprises, celles attendues dans tout discours suivi.

2) En revanche, en lien avec l'organisation et la transmission de l'information dans un discours iconique, le corpus présente des énoncés un peu particuliers en termes de structures syntaxiques et/ou référentielles :

- Du fait d'une organisation informationnelle presque entièrement gouvernée par l'iconicité de la situation de parole, le contenu et l'organisation du message dépendent plus de l'action que de réelles stratégies communicatives mises en place par le locuteur, qui n'est qu'un intermédiaire (c'est l'image qui détermine le message, pas le locuteur).

- Un bon exemple de particularité syntaxique se trouve au niveau de l'utilisation massive dans le corpus de la séquence préposition + référent en tant que syntagme autonome. Dans ce cas, le référent est introduit dans un syntagme censé être un dépendant (la préposition permet de poser une relation entre un constituant externe et ce qu'elle introduit) mais qui est utilisé par le locuteur avec le statut d'élément autonome, d'énoncé.

- On retrouve la même tendance à plus focaliser l'agent que l'action avec les séquences d'activations et de réactivations de référents qui suivent le ballon et au sein desquelles aucune expression linguistique ne renvoie à l'action, qui reste implicite.

Dans le prolongement de ce travail, il serait intéressant de considérer plus en détail le niveau prosodique, en essayant d'évaluer, entre autres, les relations qui peuvent exister entre les marques prosodiques et le degré d'activation des référents ou entre ces marques et le type d'expressions référentielles et/ou les structures syntaxiques dans lesquelles elles sont impliquées...

\section{Bibliographie}

Baumann S., Riester A. (2010), Annotating Information Status in Spontaneous Speech, in Proceedings of the Fifth International Conference on Speech Prosody. Chicago.

Blanche-Benveniste C. (1997), Macro-Syntaxe (chap.5), In C. Blanche-Benveniste, Approches de la langue parlée en français, Paris/Gap : Ophrys, 111-123.

Boulakia, G., Mathon, C. (2009), Mise en évidence de l'iconicité prosodique dans le commentaire sportif, Communication orale, ProsIco, 9-10 Avril 2009, Rouen.

Bres, J. (2007), Le discours médiatique sportif aux prises avec le temps verbal, in Broth, M., Forsgren, M., Norén, C. et Sullet-Nylander, F. (eds.), Le français parlé dans les médias, Actes du colloque de l'Université de Stockholm, 8-12 juin 2005, Acta Universitatis stolckholmiensis, Stockholm, 83-96.

Chafe, W. (1976), Givenness, contrastiveness, definiteness, subjects, topics and point of view, In C.N. Li (ed.). Subject and topic. New-York: Academic press, 27-55. 
Deulofeu, J. (1999), Les commentaires sportifs constituent-ils un "genre", au sens linguistique du terme ?, in Bilger, M. (ed.), Actes du Colloque Questions de méthode dans la linguistique sur corpus, Perpignan, 9-11 Mai 98. Paris : Champion.

Lambrecht, K. (1988), Presentational cleft constructions in spoken French, In J. Haiman \& S.A. Thompson (eds.), Clause combining in Grammar and Discourse. Amsterdam / Philadelphia : John Benjamins, 135-179.

Lortal, G., Mathon, C. (2008), Motion and Emotion or how to align emotional cues with game actions, in Proceedings of EMOT Worshop, LREC 2008, Marrakech.

Martin, P. (2000), WinPitch 2000: a tool for experimental phonology and intonation research, in Proceedings of the Prosody 2000 Workshop, Kraków, Pologne, 2-5 October 2000.

Martin, P. (2009), Intonation du français, Armand Colin.

Prince, E.F. (1981), Toward a Taxonomy of Given-New Distinction. In P. Cole (ed.), Radical Pragmatics. NewYork : Academic press, 223-255. 